ARTICLE

\title{
A mass spectrometric method for in-depth profiling of phosphoinositide regioisomers and their disease- associated regulation
}

Shin Morioka1,2,8, Hiroki Nakanishi3,8, Toshiyoshi Yamamoto1,2, Junya Hasegawa (iD 1,2, Emi Tokuda (i) 1,2, Tomoya Hikita4, Tomoko Sakihara, ${ }^{1,2}$, Yuuki Kugii,2, Chitose Oneyama (1) ${ }^{4}$, Masakazu Yamazaki (1) ${ }^{5}$, Akira Suzuki ${ }^{6}$, Junko Sasaki ${ }^{1,7,9 凶}$ \& Takehiko Sasaki (i] $1,2,9 凶$

Phosphoinositides are a family of membrane lipids essential for many biological and pathological processes. Due to the existence of multiple phosphoinositide regioisomers and their low intracellular concentrations, profiling these lipids and linking a specific acyl variant to a change in biological state have been difficult. To enable the comprehensive analysis of phosphoinositide phosphorylation status and acyl chain identity, we develop PRMC-MS (Phosphoinositide Regioisomer Measurement by Chiral column chromatography and Mass Spectrometry). Using this method, we reveal a severe skewing in acyl chains in phosphoinositides in Pten-deficient prostate cancer tissues, extracellular mobilization of phosphoinositides upon expression of oncogenic PIK3CA, and a unique profile for exosomal phosphoinositides. Thus, our approach allows characterizing the dynamics of phosphoinositide acyl variants in intracellular and extracellular milieus.

\footnotetext{
${ }^{1}$ Department of Biochemical Pathophysiology, Medical Research Institute, Tokyo Medical and Dental University, Tokyo 113-8510, Japan. ${ }^{2}$ Department of Lipid Biology, Graduate School of Medical and Dental Sciences, Tokyo Medical and Dental University, Tokyo 113-8510, Japan. ${ }^{3}$ Research Center for Biosignal, Akita University, Akita 010-8543, Japan. ${ }^{4}$ Division of Cancer Cell Regulation, Aichi Cancer Center Research Institute, Chikusa-ku, Nagoya 464-8681, Japan. ${ }^{5}$ Department of Cell Biology and Morphology, Akita University Graduate School of Medicine, Akita 010-8543, Japan. ${ }^{6}$ Division of Molecular and Cellular Biology, Graduate School of Medicine, Kobe University, Kobe 650-0017, Japan. ${ }^{7}$ Department of Life Science and Technology, Graduate School of Medical and Dental Sciences, Tokyo Medical and Dental University, Tokyo 113-8510, Japan. ${ }^{8}$ These authors contributed equally: Shin Morioka, Hiroki Nakanishi.

${ }^{9}$ These authors jointly supervised this work. Junko Sasaki, Takehiko Sasaki. ${ }^{凶}$ email: jsaspip@tmd.ac.jp; tsasaki.pip@mri.tmd.ac.jp
} 
P hosphoinositides comprise one of the most functionally versatile membrane lipid families involved in human health and disease $\mathrm{e}^{1,2}$. The base structure of all phosphoinositides contains phosphatidylinositol (PI), which is made up of an inositol head group and two long-chain fatty acids linked to a glycerol backbone (Fig. 1a). Combinatorial phosphorylation of residues in the PI head group gives rise to seven other phosphoinositide classes, namely $\mathrm{PI}(3) \mathrm{P}, \mathrm{PI}(4) \mathrm{P}, \mathrm{PI}(5) \mathrm{P}, \mathrm{PI}(3,4) \mathrm{P}_{2}$, $\mathrm{PI}(3,5) \mathrm{P}_{2}, \mathrm{PI}(4,5) \mathrm{P}_{2}$, and $\mathrm{PI}(3,4,5) \mathrm{P}_{3}$. These lipids spatiotemporally control the activities of many proteins possessing phosphoinositide-binding motifs. These motifs can bind to various phosphoinositides with differing affinities to regulate numerous physiological processes in cells ${ }^{3}$.

Genetic alterations in phosphoinositide-metabolizing enzymes, including kinases, phosphatases, lipases, and acyltransferases, have been implicated in the pathogenesis of various diseases ${ }^{4,5}$. However, it is unclear whether or how these genetic changes alter phosphoinositide configurations; that is, it is not known if the composition of various phosphoinositide classes and their acyl variants differ in the cells or tissues of diseased individuals compared to healthy individuals. Part of the challenge in addressing this issue lies in the current difficulties in measuring specific phosphoinositide acyl variants.

Measurements of cellular phosphoinositides have traditionally been carried out by anion-exchange HPLC of lipid extracts prepared from cultured cells that were metabolically labeled with $\left[{ }^{3} \mathrm{H}\right]$ inositol and/or $\left[{ }^{32} \mathrm{P}\right]$ phosphorus ${ }^{6}$. Although this method effectively quantifies the relative levels of the eight phosphoinositide classes, it cannot easily be applied to clinical samples or pathological samples from experimental animals. In addition, this approach cannot provide any information on differences in the acyl chains of phosphoinositide species because the hydrophobic moiety has to be removed before separation by anion-exchange chromatography. Recently, Clark et al. devised a significant improvement in phosphoinositide measurement by combining reverse-phase HPLC with electrospray ionization-tandem mass spectrometry (ESI-MS/MS) ${ }^{7}$. This method enables the quantification of phosphoinositide molecular species in a spectrum of biological samples, including those not amenable to radioisotope labeling. Ghosh et al. have also described a mass spectrometry method based on the use of recombinant specific lipid-kinases to in vitro phosphorylate $\mathrm{PI} 3 \mathrm{P}$ and $\mathrm{PI}$ P into $\mathrm{PI}(3,5) \mathrm{P}_{2}$ and $\mathrm{PI}(4,5)$ $\mathrm{P}_{2}$, respectively ${ }^{8}$. However, it still remains a hurdle to simultaneously quantitate the acyl variants of all individual regioisomers of PI-monophosphate (PI3P/PI4P/PI5P) and -bisphosphate $\left[\mathrm{PI}(3,4) \mathrm{P}_{2} / \mathrm{PI}(3,5) \mathrm{P}_{2} / \mathrm{PI}(4,5) \mathrm{P}_{2}\right]$ as well as $\mathrm{PI}$ and $\mathrm{PI}(3,4,5) \mathrm{P}_{3}$ in a

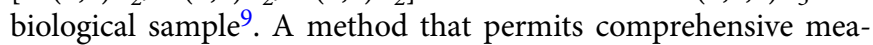
surement of the entire set of phosphoinositides would advance our understanding of how all these lipids are dynamically engaged to control cell functions.

Here we report a method for Phosphoinositide Regioisomer Measurement by Chiral column chromatography and Mass Spectrometry (PRMC-MS) that solves the above problem and can be applied to many types of biological samples. PRMC-MS reveals that phosphoinositide acyl variants are differentially accumulated in normal mouse prostate and Pten-deficient prostate cancer tissues and further identifies extracellular mobilization of the phosphoinositide variants.

\section{Results}

An HPLC-MS-based method to measure the eight phosphoinositide classes at the acyl variant level. The general workflow of our PRMC-MS method to measure phosphoinositide acyl variants (defined by the number of carbons and the degree of unsaturation in the hydrocarbon chains) is depicted in Fig. $1 \mathrm{~b}$. We devised this method to circumvent two confounding issues: (1) The anion-exchange column chromatography approach previously introduced for the separation of glycerophosphoinositol phosphates (the deacylated products of phosphoinositides) cannot be easily adapted for ESI-MS/MS due to the requirement for a non-volatile phosphate buffer. (2) Reverse-phase columns do not discriminate among phosphoinositide regioisomers. We, therefore, set out to adapt chiral column chromatography and took advantage of the mobile phases of acetonitrile and methanol. Synthetic phosphoinositides (heptadecanoyl-arachidonyl; C37:4 molecular species) were used as standard analytes whose phosphate groups had been methylated by a reaction with trimethylsilyl (TMS) diazomethane ${ }^{7}$. The derivatized phosphoinositides were mixed in various combinations, separated on a stationary phase where a chiral selector was immobilized to silica gel matrices, and detected with an in-line triple quadrupole mass
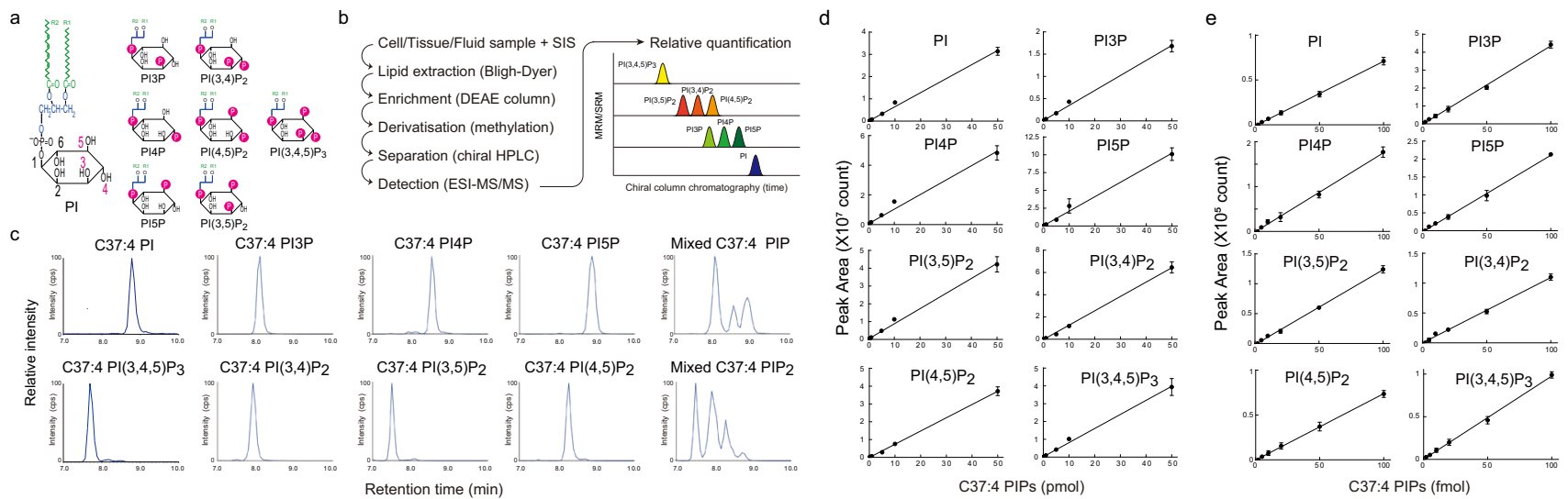

Fig. 1 Phosphoinositide regioisomer measurement by PRMC-MS. a Schematic structures of the eight phosphoinositide classes. Note that phosphatidylinositol mono- and bisphosphate (PIP and PIP 2 ) classes each contain three regioisomers. b Scheme depicting the general workflow of PRMCMS. SIS, surrogate internal standard lipids. c Mass spectra from PRMC-MS analyses of C37:4 (1-heptadecanoyl-2-eicosatetraenoyl) standard chemicals representing each of the eight phosphoinositide classes. Mixed, a mixture of PIP or PIP 2 regioisomers. $\mathbf{d}$, e Linear regression analyses of peak area vs pmol (d) and fmol (e) concentrations of the indicated phosphoinositides. Amounts of standard phosphoinositides analyzed were 0.002, 0.005, 0.01, 0.02, 0.05, $0.1,0.2,0.5,1,5,10,50 \mathrm{pmol}$ in $\mathbf{d}$ and 2, 5, 10, 20,50, $100 \mathrm{fmol}$ in e (see also Supplementary Table 1). The C37:4 (1-heptadecanoyl-2-eicosatetraenoyl) molecular species of each phosphoinositide class was used. Data were the mean \pm SD ( $n=4$ technical replicates). Source data are provided as a Source Data file. 
spectrometer using the "multiple reaction monitoring" (MRM) technique ${ }^{10}$. During trial-and-error testing of chromatography columns, the chiral selector cellulose tris(3,5-dichlorophenylcarbamate) was found to successfully separate the three PI-monophosphate (PIP) and three PI-bisphosphate $\left(\mathrm{PIP}_{2}\right)$ regioisomers (Fig. 1C and Supplementary Fig. 1). PI and PItrisphosphate $\left[\mathrm{PI}(3,4,5) \mathrm{P}_{3}\right]$ could also be analyzed in the same run using different MRM transitions. Thus, our method can simultaneously measure all eight classes of phosphoinositides in a single sample. Repeatable quantification was confirmed by 50 successive measurements of C37:4 phosphoinositide standards with a coefficient of variation (CV) values of $4.9-12.0 \%$ (Supplementary Table 1). Good linear correlations were obtained between the ion-current peak areas and chemical amounts ranging from sub-fmol to 50 pmol of the phosphoinositide standards (Fig. 1d, e and Supplementary Table 1). These data establish that our PRMC-MS approach has a wide dynamic range and a low detection limit for each phosphoinositide class.

Application of PRMC-MS to intracellular phosphoinositide measurement. Next, we examined whether our PRMC-MS method was able to measure phosphoinositides in lipid extracts prepared from HEK293T cells. Considering the minor proportion of phosphoinositides to total phospholipids in cellular lipid extracts, an acidic lipid-enriched fraction was obtained using an anion-exchange column prior to the methylation reaction to prevent ion suppression in mass spectrometry. PRMC-MS successfully distinguished three regioisomers each of PIP and $\mathrm{PIP}_{2}$ in lipid extracts of HEK293T cells, and pre-concentration by passage over DEAE Sepharose enhanced the peak intensities of all phosphoinositides (Supplementary Fig. 2a). The yields [peak area with pre-concentration divided by peak area without preconcentration (triplicate average)] were $20.2 \%$ (PI), $92.2 \%$ (PI3P), 81.9\% (PI4P), 89.6\% (PI5P), 167\% (PI(3,4) $\left.\mathrm{P}_{2}\right), 128 \%$ $\left(\mathrm{PI}(3,5) \mathrm{P}_{2}\right), 134 \%\left(\mathrm{PI}(4,5) \mathrm{P}_{2}\right)$, and $113 \%\left(\mathrm{PI}(3,4,5) \mathrm{P}_{3}\right)$ when determined using synthetic C37:4 phosphoinositides. This enrichment procedure was especially effective for measuring minor phosphoinositide classes such as $\mathrm{PI} 3 \mathrm{P}, \mathrm{PI}$ P, $\mathrm{PI}(3,4) \mathrm{P}_{2}$, $\mathrm{PI}(3,5) \mathrm{P}_{2}$, and $\mathrm{PI}(3,4,5) \mathrm{P}_{3}$, as well as some minor acyl variants of $\mathrm{PI}, \mathrm{PI} 4 \mathrm{P}$, and $\mathrm{PI}(4,5) \mathrm{P}_{2}$, although it failed to steadily detect previously reported $\mathrm{PIP}$ and $\mathrm{PIP}_{2}$ variants containing highly polyunsaturated acyl chains (e.g., 40:7, 36:8, 40:9, and 42:10) ${ }^{11}$. Preconcentration was also helpful in reducing the maintenance/ cleaning of the mass spectrometer because the amount of lipid loaded could be kept to a minimum.

Supplementary Table 2 lists the MRM transitions (pairs of $m / z$ values of precursor ions and fragment/diacylglycerol ions) used for the identification and quantification of each phosphoinositide acyl variant. Endogenous concentrations of the acyl variants in each class were estimated based on those of the class-matched C37:4 phosphoinositides (surrogate internal standards; SIS) added to the cell pellets before lipid extraction, allowing the relative quantification of intracellular acyl variants. Practically, the chromatographic peak area of an endogenous phosphoinositide was divided by the area of the class-matched SIS (Supplementary Fig. 3). As shown in Fig. 2a, PI, PI3P, PI4P, $\mathrm{PI}(3,5) \mathrm{P}_{2}, \mathrm{PI}(4,5) \mathrm{P}_{2}$, and $\mathrm{PI}(3,4,5) \mathrm{P}_{3}$ were readily detected in resting HEK293T cells. Approximately linear correlations were obtained between cell numbers and the amounts of the phosphoinositide acyl variants identified (Supplementary Fig. 4 and Supplementary Table 3 ). We did not find the peaks corresponding to any acyl variants of $\mathrm{PI}(5) \mathrm{P}$ and $\mathrm{PI}(3,4) \mathrm{P}_{2}$ in resting HEK293T cells probably because these lipids were present below the detection limit of PRMC-MS under normal culture conditions. To test whether PRMC-MS can detect cellular PI(5)P, we analyzed HEK293T cells engineered to overexpress IpgD [a Shigella flexneri virulence factor that converts $\mathrm{PI}(4,5) \mathrm{P}_{2}$ to $\left.\mathrm{PI}_{5} \mathrm{P}^{12}\right]$. In parallel, to detect intracellular $\mathrm{PI}(3,4) \mathrm{P}_{2}$, we treated non-transfected HEK293T cells with hydrogen peroxide $\left(\mathrm{H}_{2} \mathrm{O}_{2}\right)^{13}$. We found that IpgD expression led to an increase in PI5P, whereas $\mathrm{H}_{2} \mathrm{O}_{2}$ treatment increased $\mathrm{PI}(3,4) \mathrm{P}_{2}$ (Fig. 2a). Thus, PRMC-MS allows the simultaneous measurement of all 8 phosphoinositide classes at the acyl variant level in cell extracts.

Vps34 and the class II PI3Ks produce PI3P, and various PI3P pools are proposed to be involved in distinct cellular processes, including autophagic programs ${ }^{14}$. PIK-III is a potent and selective Vps34 inhibitor that binds a unique hydrophobic pocket not present in class I/II PI3Ks ${ }^{15}$. PIK-III decreased PI3P by $\sim 40 \%$ in HeLa cells, indicating that PRMC-MS can differentiate between Vps34-dependent and -independent PI3P pools within cells (Fig. 2b). In addition, there were smaller but statistically significant decreases in $\mathrm{PI}(3,5) \mathrm{P}_{2}$ (a phosphorylated derivative of PI3P) and PI4P levels between control and PIK-III treated cells. Similarly, previous work has shown that epidermal growth factor (EGF) induces the accumulation of $\mathrm{PI}(3,4) \mathrm{P}_{2}$ and $\mathrm{PI}(3,4,5)$ $\mathrm{P}_{3}$ in a class I PI3K-dependent manner ${ }^{16}$. Using PRMC-MS to examine EGF-treated PC3 cells, we found that $\mathrm{PI}(3,4) \mathrm{P}_{2}$ and $\mathrm{PI}(3,4,5) \mathrm{P}_{3}$ were increased while $\mathrm{PI}(4,5) \mathrm{P}_{2}$ (the primary substrate of class I PI3Ks) was decreased (Fig. 2c). We also confirmed that these responses were abolished by the pan-class I PI3K inhibitor copanlisib $^{17}$. Thus, PRMC-MS is exquisitely sensitive and highly useful for the detection of small but important changes in intracellular phosphoinositide levels in response to environmental cues.

Distorted phosphoinositide profile in prostate cancer tissue from Pten-deficient mice. In vivo measurement of phosphoinositides has been limited due to the necessity of radioisotope labeling of animals, which is hazardous and labor-intensive. We exploited a cancer mouse model to test our PRMC-MS method in tissue samples. The phosphatase PTEN, whose primary substrate is $\operatorname{PI}(3,4,5) \mathrm{P}_{3}$, is one of the most frequently altered tumor suppressors across a variety of cancer types ${ }^{18}$. We used PRMC-MS to analyze the phosphoinositide profiles of prostate tissues isolated from healthy wild-type (WT) C57BL/6 mice and those from prostate tumor-bearing Pten-deficient (PbCre4-Pten flox/flox 119,20 ) mice (Fig. 3). Consistent with a previous study employing HPLCMS analysis and immunohistochemistry ${ }^{16}$, levels of $\mathrm{PI}(3,4,5) \mathrm{P}_{3}$ and $\mathrm{PI}(3,4) \mathrm{P}_{2}$ were heightened in in vivo samples of the prostates of Pten-deficient mice. In addition, PI3P was increased while PI4P remained unchanged in Pten-deficient prostates. Intriguingly, we observed a dramatic skewing in the acyl variant composition of $\mathrm{PI}(3,4,5) \mathrm{P}_{3}$ in the Pten-deficient prostates. For instance, levels of $\mathrm{C} 32: 0$ and $\mathrm{C} 34: 1 \mathrm{PI}(3,4,5) \mathrm{P}_{3}$ were increased $\sim 50$-fold in the tumor samples compared to controls, whereas $\mathrm{C} 38: 4 \mathrm{PI}(3,4,5) \mathrm{P}_{3}$ (the most abundant acyl variant in normal prostate) was almost unchanged. The fatty acyl profile of $\mathrm{PI}(3,4)$ $\mathrm{P}_{2}$ in cancerous prostate tissue resembled that of $\mathrm{PI}(3,4,5) \mathrm{P}_{3}$, supporting the previously described notion that $\mathrm{PI}(3,4) \mathrm{P}_{2}$ in tumors is produced mainly through $5^{\prime}$-dephosphorylation of $\mathrm{PI}(3,4,5) \mathrm{P}_{3}{ }^{16}$. These data demonstrate that not only the quantity but also the composition of $\mathrm{PI}(3,4) \mathrm{P}_{2}$ and $\mathrm{PI}(3,4,5) \mathrm{P}_{3}$ are disturbed in Pten-deficient cancer tissue. Our results further suggest that the detailed profiling of phosphoinositide acyl variants may reveal molecular signatures useful for cancer stratification.

Extracellular mobilization of phosphoinositides. The question of the existence of "extracellular" phosphoinositides has also been difficult to address using existing protocols, and so we investigated whether our PRMC-MS method could be extended to 

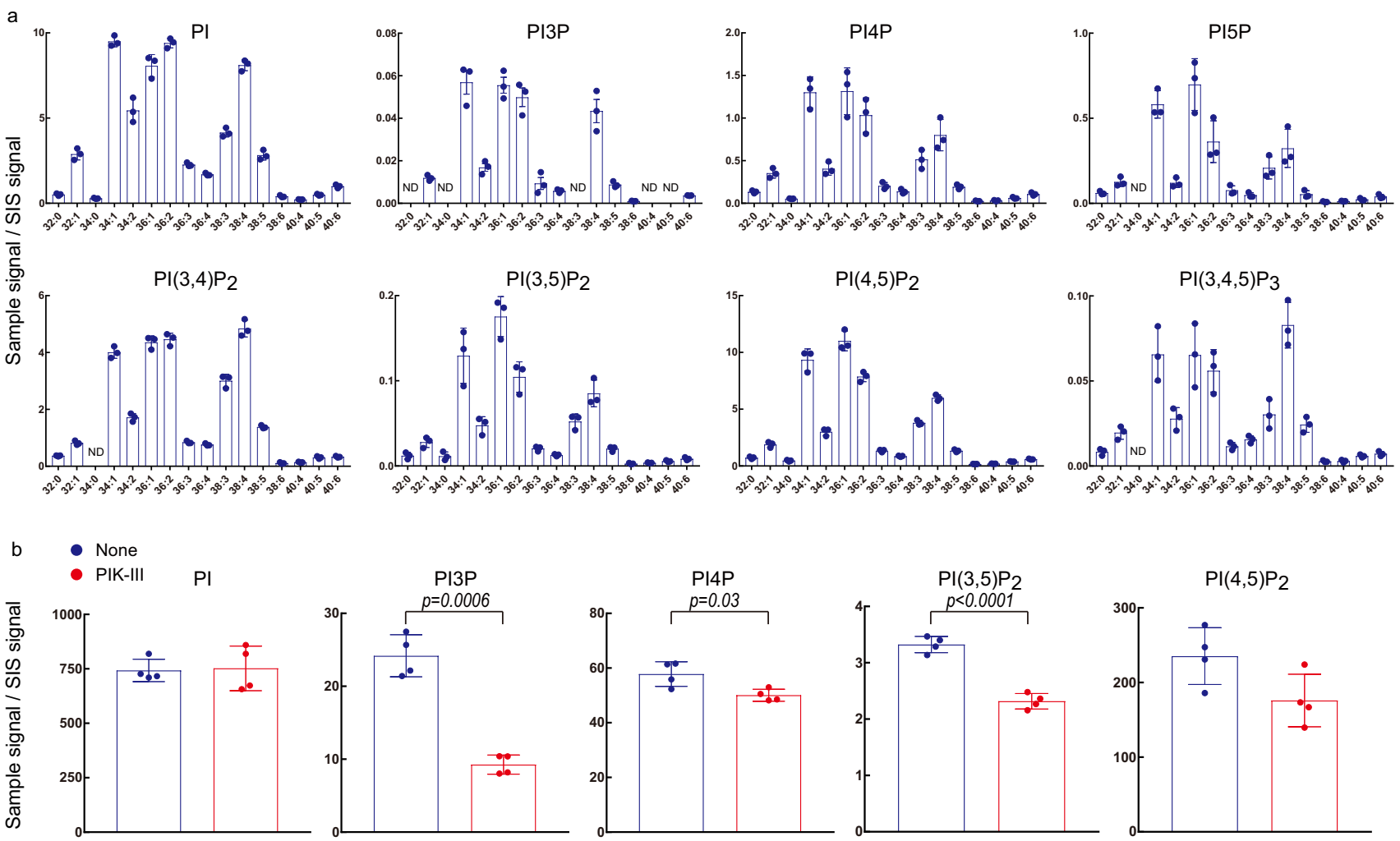

$\mathrm{PI}(4,5) \mathrm{P} 2$

c
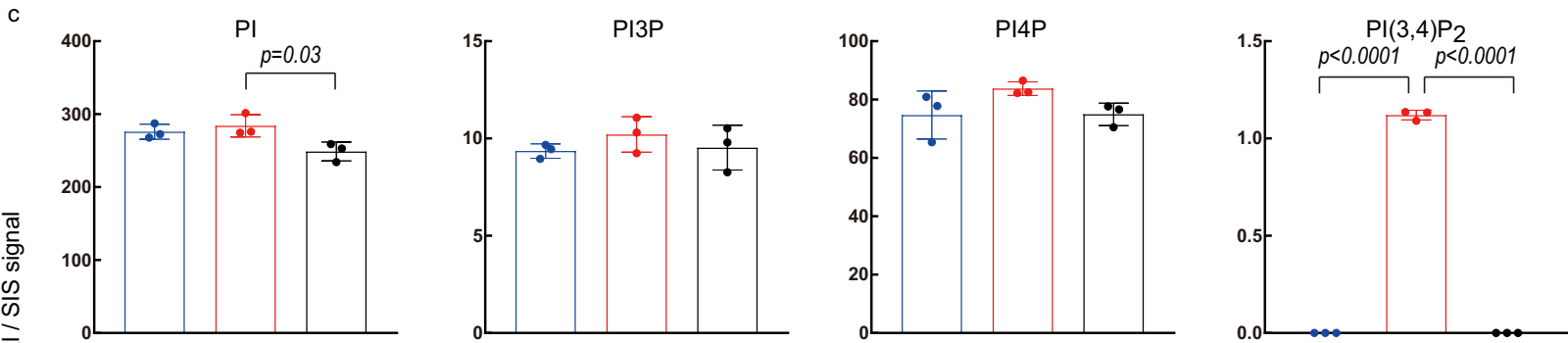

$\mathrm{PI}(3,5) \mathrm{P}_{2}$
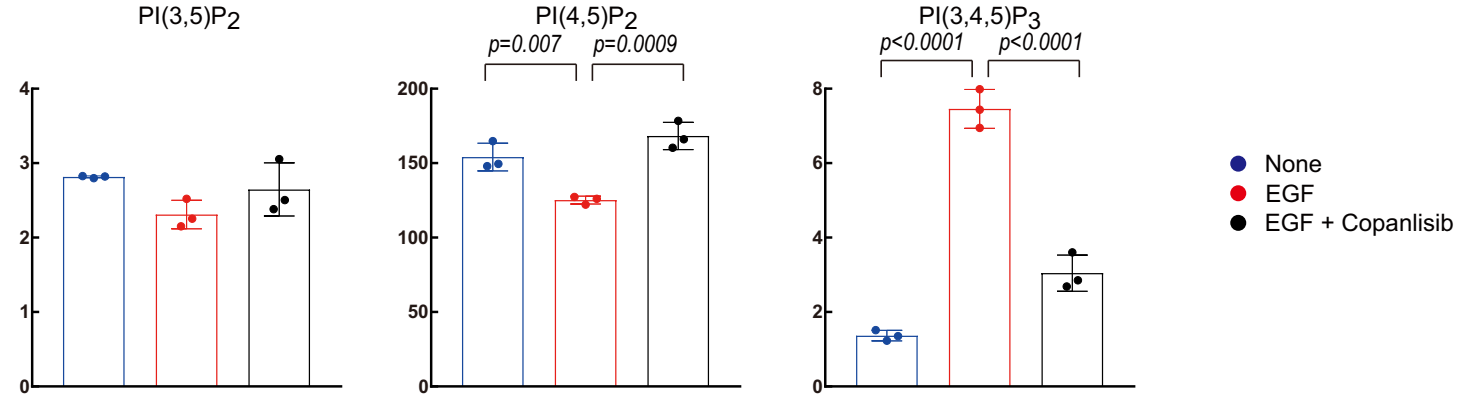

Fig. 2 Application of PRMC-MS to phosphoinositide measurement in cell lines. a Levels of the indicated phosphoinositide acyl variants in HEK293T cells. $\mathrm{PI}, \mathrm{PI} \mathrm{P}, \mathrm{PI} 4 \mathrm{P}, \mathrm{PI}(3,5) \mathrm{P}_{2}, \mathrm{PI}(4,5) \mathrm{P}_{2}$, and $\mathrm{PI}(3,4,5) \mathrm{P}_{3}$ were assessed in mock-transfected cells. PI5P was assessed in cells overexpressing IpgD. PI(3,4) $\mathrm{P}_{2}$ was assessed in non-transfected cells treated with $1 \mathrm{mM} \mathrm{H}_{2} \mathrm{O}_{2}$ for $5 \mathrm{~min}$. Data were the mean $\pm \mathrm{SD}$ ( $n=3$ biologically independent samples). ND not determined. $\mathbf{b}$ Phosphoinositide measurements in HeLa cells treated with vehicle (control) or $5 \mu \mathrm{M} \mathrm{PIK}-\mathrm{III}$ for $2 \mathrm{~h}$. $\mathrm{PI} 5 \mathrm{P}, \mathrm{PI}(3,4) \mathrm{P}_{2}$, and $\mathrm{PI}(3,4,5) \mathrm{P}_{3}$ were not detected. Data were the mean \pm SD of total acyl variants of the indicated phosphoinositide classes $(n=4$ biologically independent samples), and $p$ values by two-sided Welch's $t$-test are shown. c Phosphoinositide measurements in PC3 cells that were left untreated (control), or treated with vehicle or $100 \mathrm{nM}$ copanlisib for $30 \mathrm{~min}$ prior to stimulation with $10 \mathrm{ng} / \mathrm{ml}$ EGF. PI5P was not detected. Data were the mean \pm SD of total acyl variants of the indicated phosphoinositide classes ( $n=3$ biologically independent samples). The significance was analyzed using a two-sided Dunnett's $t$-test. Source data are provided as a Source Data file.

address this issue. We successfully detected PI, PI3P, PI4P, PI(3,5) $\mathrm{P}_{2}, \mathrm{PI}(4,5) \mathrm{P}_{2}$, and $\mathrm{PI}(3,4,5) \mathrm{P}_{3}$ in culture supernatants (conditioned medium; CM) of resting HEK293T cells (Fig. 4a). To determine the effect of oncogenic phosphoinositide 3-kinase expression on extracellular phosphoinositides, we examined
HEK293T cells overexpressing various mutant PIK3CA proteins. PIK3CA gain-of-function mutations frequently occur at two hotspots: one in the helical domain (E545K) and one in the kinase domain $(\mathrm{H} 1047 \mathrm{R})^{4}$. We found that ectopic expression of these mutant PIK3CA molecules caused enormous increases in 
a
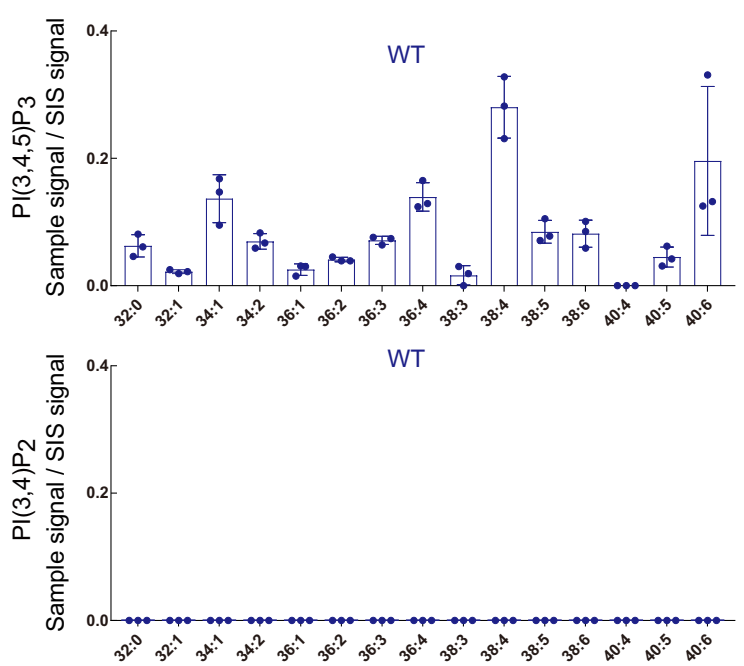

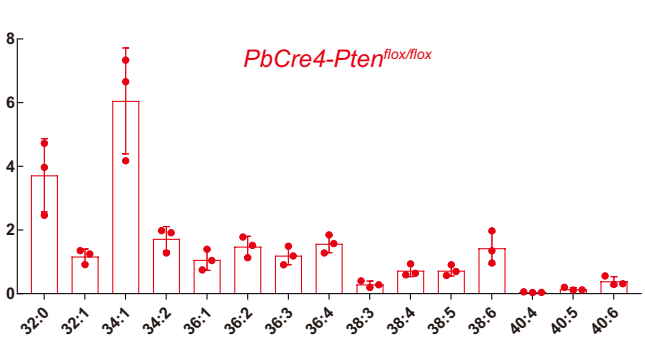

Total $\mathrm{PI}(3,4,5) \mathrm{P}_{3}$
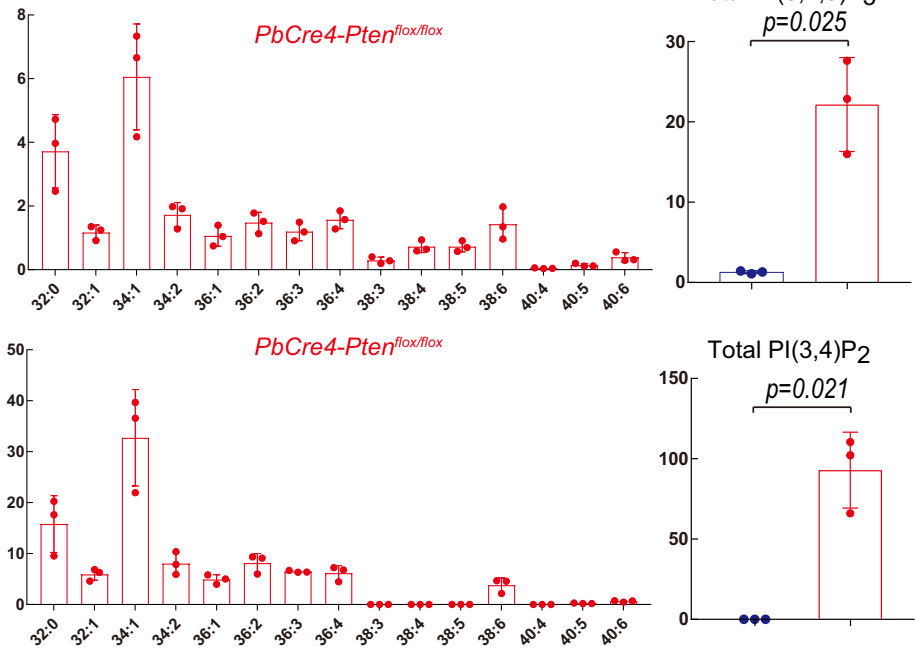

$\mathrm{b}$

WT PbCre4-Pten floxflox
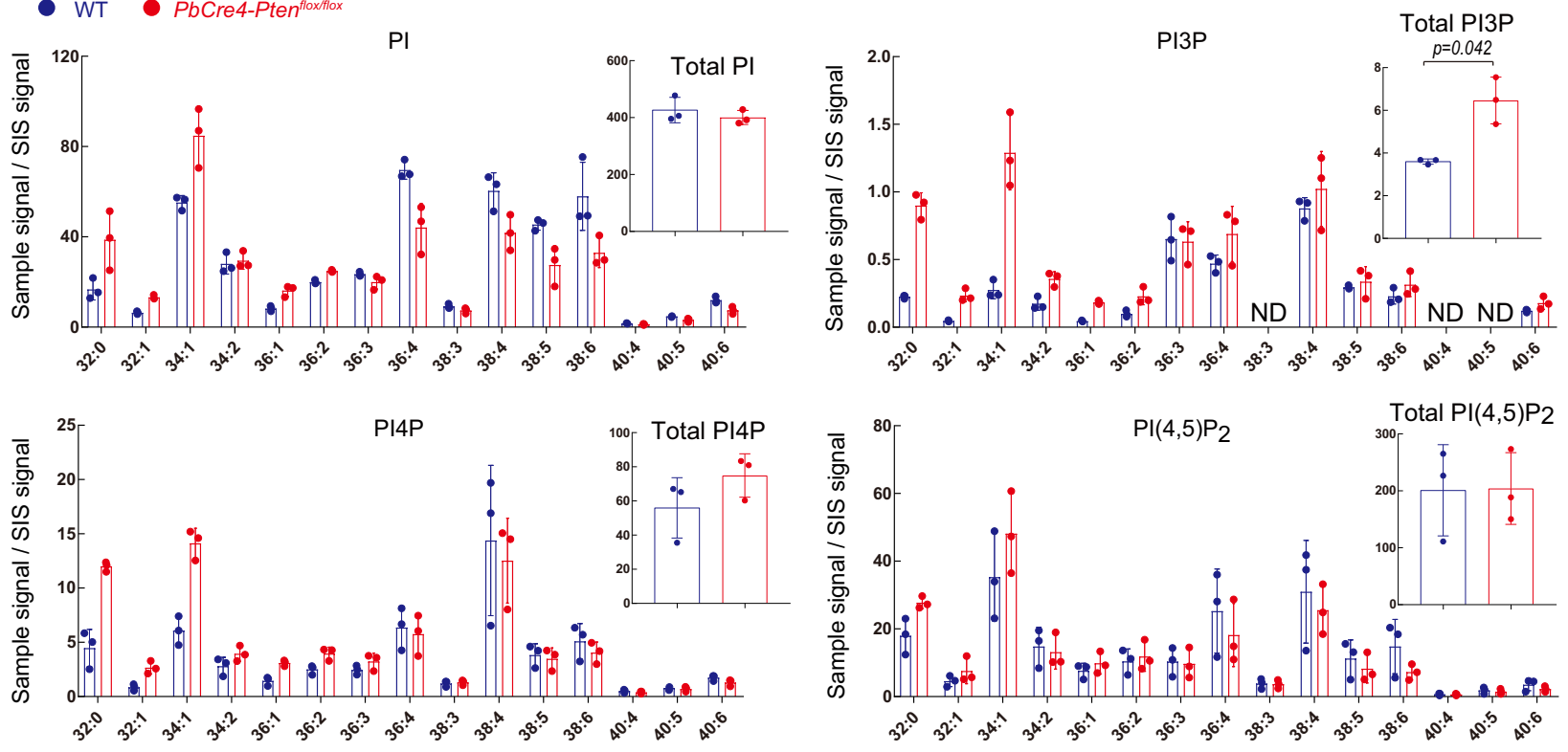

Fig. 3 Application of PRMC-MS to phosphoinositide measurement in mouse tissue. a Left and middle panels: Quantification of the indicated $\mathrm{PI}(3,4,5) \mathrm{P}_{3}$ and $\mathrm{PI}(3,4) \mathrm{P}_{2}$ acyl variants in prostate tissues from WT and prostate-specific Pten-deficient $(P b C r e 4-P$ tenflox/flox $)$ mice at 12 weeks of age $(n=3$ independent animals/group). The altered acyl chain profile in Pten-deficient cancerous tissues was confirmed in five independent experiments. Right panel: Total of the $\mathrm{PI}(3,4) \mathrm{P}_{2}$ and $\mathrm{PI}(3,4,5) \mathrm{P}_{3}$ acyl variants in the tissues in the left and middle panels. b Measurements of the indicated acyl variants and total $\mathrm{PI}$ $\mathrm{PI3P}, \mathrm{PI} 4 \mathrm{P}$, and PI(4,5) $\mathrm{P}_{2}$ in the prostate tissues from the WT (blue) and PbCre4-Ptenflox/flox (red) mice in (a) (12 weeks of age, $n=3$ ). For all panels, data were the mean \pm SD ( $n=3$ independent animals). ND not determined. The significance between WT and PbCre4-Pten flox/flox mice was analyzed using a two-sided Welch's t-test. Source data are provided as a Source Data file.

extracellular $\mathrm{PI}(3,4,5) \mathrm{P}_{3}$, with smaller or no increases in other phosphoinositide classes (Fig. 4a). The presence of extracellular phosphoinositides was not unique to HEK293T cell cultures as these lipids were detected in CM of all cell lines tested, including LNCap cells (Fig. $4 \mathrm{~b}-\mathrm{d}$ ).

Because phosphoinositides were detected in culture supernatants passed through $0.45 \mu \mathrm{m}$ pores, it was unlikely that these lipids were derived from floating dead cells. To gain insight into the origin of these extracellular phosphoinositides, we isolated exosomes (extracellular vesicles of $50-150 \mathrm{~nm}$ in size) from LNCap culture supernatants. PRMC-MS revealed that these exosomes did indeed contain substantial amounts of phosphoinositides that accounted for about $10 \%$ of total phosphoinositides in CM (Fig. 4d). The acyl variant compositions of phosphoinositides in exosomes and CM were similar to those in intracellular samples (Fig. 4c,d and Supplementary Fig. 5).

The above findings in cultured cells prompted us to examine extracellular phosphoinositides in vivo. Blood samples were collected from WT mice that had been injected with either phosphate-buffered saline (PBS; control) or bacterial lipopolysaccharide (LPS). In control mice, all phosphoinositide classes except $\mathrm{PI}(3,4) \mathrm{P}_{2}$ were detected in the plasma (Fig. 4e). LPS treatment increased plasma levels of $\mathrm{PI}$ and $\mathrm{PI}(3,4,5) \mathrm{P}_{3}$. These data establish that PRMC-MS can be applied to blood samples to track phosphoinositide signatures potentially related to inflammatory disease states. In the future, it will be interesting to investigate the specific biological activity of the extracellular form of each class of phosphoinositide. 


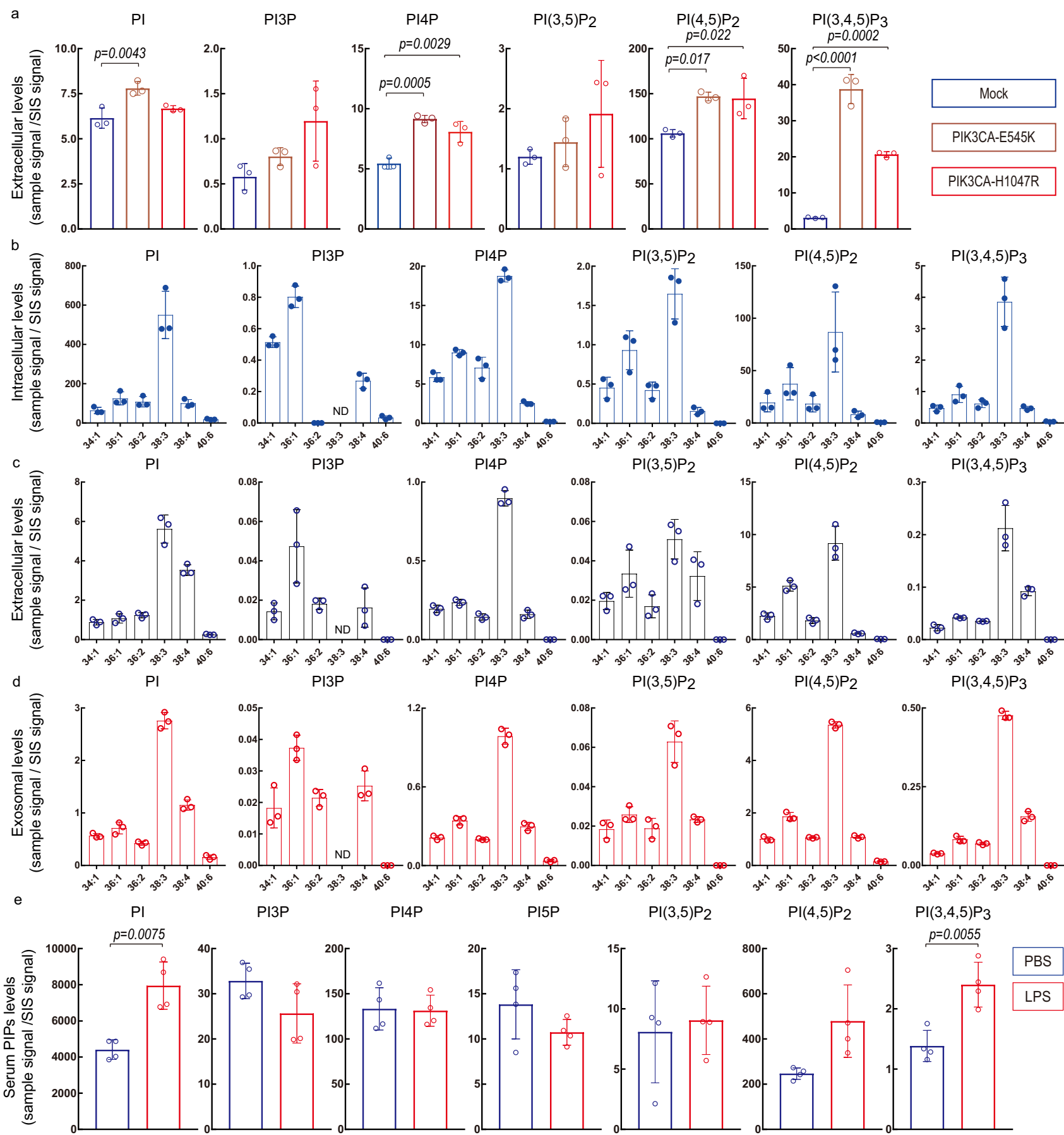

Fig. 4 Application of PRMC-MS to phosphoinositide measurement in liquid samples. a Quantification of the indicated phosphoinositides in CM of HEK293T cells transfected with either control expression vector (Mock) or vector expressing either mutant E545K or H1047R PIK3CA. CM was collected at $24 \mathrm{~h}$ post-transfection. Data were the mean \pm SD ( $n=3$ biologically independent samples) and representative of three independent experiments. Statistical analysis was performed using a two-sided Dunnett's $t$-test. b-d Quantification of the indicated phosphoinositide acyl variants in $\mathbf{b}$ cell pellets, $\mathbf{c}$ $\mathrm{CM}$, and $\mathbf{d}$ exosomes derived from LNCap cell cultures. PI5P and PI $(3,4) \mathrm{P}_{2}$ were not detected. Data were the mean \pm SD $(n=3$ biologically independent samples). e Quantification of the indicated phosphoinositides in plasma from WT mice that were intraperitoneally injected with either vehicle (PBS) or $50 \mathrm{mg} / \mathrm{ml}$ lipopolysaccharide (LPS) and analyzed after $2 \mathrm{~h} . \mathrm{PI}(3,4) \mathrm{P}_{2}$ was not detected in either case. Data were the mean \pm SD of total acyl variants of the indicated phosphoinositide classes ( $n=4$ biologically independent samples). Statistical analysis was performed using two-sided unpaired Welch's $t$-test. Source data are provided as a Source Data file.

\section{Discussion}

The PRMC-MS approach described here enables the comprehensive analysis of phosphoinositide acyl variants in various types of biological samples, including cultured cells, CM, exosomes, isolated tissues, and blood. We have shown that PRMC-MS can be used to highlight previously unrecognized disturbances of phosphoinositide fatty acyl profiles in cancerous tissue and to monitor the extracellular mobilization of phosphoinositides.

The physiological importance of molecularly heterogeneous acyl chain compositions of phosphoinositides in a cell has long 
been an enigma. Further studies should determine the cancer cell characteristics affected by the observed skewing in phosphoinositide acyl chains in the Pten-deficient prostate. An attractive hypothesis is that different acyl variants confer different proteinbinding properties due to the influence of the hydrophobic moiety on the interaction of the lipid with proteins at the cell membrane. Such interactions could potentially activate a signaling pathway that favors cancer cell growth and survival and emerge as a target for the therapy of cancer. We previously analyzed phosphoinositide acyl variants in prostate cancer tissues from 16 surgical patients using reverse-phase chromatographytandem mass spectrometry; however, we did not find a similar acyl chain distortion as in Pten-deficient mouse samples. Investigations using a larger number and more diverse types of human cancers should be conducted to elucidate the pathological relevance of the fluctuations in each acyl variant as well as their interaction with changes in oncogenes and tumor suppressor genes such as PIK3CA, $\mathrm{K}$-, H-RAS, and PTEN.

Another finding of our study is its demonstration of the existence of extracellular phosphoinositides. Extracellular phosphoinositides were mobilized, at least in part, in the form of the exosomes. These results indicate that cells actively secrete phosphoinositides, which may in turn act on neighboring cells. Our finding that cellular activation by oncogenic PI3K mutant expression or LPS administration changes the extracellular phosphoinositide profile raises the possibility that autocrine/ paracrine secretion of phosphoinositides may play a role in the pathogenesis of cancers and inflammatory diseases. Thus, PRMCMS may pave the way for a field of study on the pathophysiological functions of phosphoinositides in the extracellular environment.

While this study was under review, Li and Lämmerhofer reported a method for the measurement of phosphoinositide regioisomers ${ }^{21}$. It also took advantage of chiral chromatography for regioisomer separation as PRMC-MS, though the chiral selector used was cellulose tris(3,5-dimethylphenylcarbamate). In addition, a different data acquisition method, sequential window acquisition of all theoretical mass spectra (SWATH), was adopted in the method.

A growing body of evidence demonstrates that alterations to genes encoding phosphoinositide kinases and phosphatases (which shape phosphorylation patterns) and lysophospholipid acyltransferases (which shape the acyl chains) are associated with cellular dysfunction and disease ${ }^{9,22}$. The use of PRMC-MS to evaluate phosphoinositide signatures at the acyl variant level in tissue and liquid biopsies in a particular disease context may reveal biomarkers suitable for a wide variety of clinical applications. Such applications may then facilitate rational drug development strategies based on the devising of a therapeutic agent that pinpoints a specific pathogenic phosphoinositide acyl variant.

\footnotetext{
Methods

Lipids and reagents. 1-heptadecanoyl-2- (5Z, 8Z, 11Z, 14Z-eicosatetraenoyl)-snglycero-3-phospho-(1-myo-inositol-3,4,5-trisphosphate) [17:0/20:4 $\left.\mathrm{PI}(3,4,5) \mathrm{P}_{3}\right]$ 17:0/20:4 PI(3,4) $\mathrm{P}_{2}, 17: 0 / 20: 4$ PI(3,5) $\mathrm{P}_{2}, 17: 0 / 20: 4$ PI(4,5) $\mathrm{P}_{2}, 17: 0 / 20: 4$ PI3P, 17:0/ 20:4 PI4P, 17:0/20:4 PI5P, 17:0/20:4 PI, 1-stearoyl-2- (5Z, 8Z, 11Z, 14Z-eicosatetraenoyl)-sn-glycero-3-phospho-(1-myo-inositol-3,4,5-trisphosphate) [18:0/20:4 $\left.\mathrm{PI}(3,4,5) \mathrm{P}_{3}\right], 18: 0 / 20: 4 \mathrm{PI}(3,4) \mathrm{P}_{2}, 18: 0 / 20: 4 \mathrm{PI}(3,5) \mathrm{P}_{2}, 18: 0 / 20: 4 \mathrm{PI}(4,5) \mathrm{P}_{2}, 18: 0 / 20: 4$ PI3P, 18:0/20:4 PI4P, 18:0/20:4 PI5P, 18:0/20:4 PI, and 1-heptadecanoyl-2- (5Z, 8Z, 11Z, 14Z-eicosatetraenoyl)-sn-glycero-3-phospho-L-serine were purchased from Avanti. PI(4,5) $\mathrm{P}_{2}$ (8:0/8:0) and copanlisib was from Cayman. Methanol, chloroform, acetonitrile, ammonium acetate, ultrapure water, hydrochloric acid, 28\% aqueous ammonia, and glacial acetic acid were all HPLC grade or MS grade and purchased from Fujifilm Wako Pure Chemical. Trimethylsilyl diazomethane was from Tokyo Kasei. DEAE Sepharose Fast Flow was from GE Healthcare. Glass tubes $(12 \mathrm{~mL}$ ) were from Corning. DMEM and bovine serum albumin (fatty acid-
} free) were from Nacalai Tesque. Hydrogen peroxide solution $(30 \%)$ was from
Fujifilm Wako. Chiral HPLC columns were from DAICEL and C18 columns were from GL Sciences. Recombinant human EGF was from PeproTech. Lipopolysaccharide (LPS; Escherichia coli O111: B4) was from Sigma-Aldrich. PIK-III was from Selleck Chemicals.

Mice. All mice were under a normal light/dark condition $(12 \mathrm{~h}$ light $/ 12 \mathrm{~h}$ dark cycle). The animal colony was kept at $20-26^{\circ} \mathrm{C}$ with $40-60^{\circ} \mathrm{C}$ humidity. The generation and genotyping of PbCre4-Pten flox/flox mice have been previously described ${ }^{19,20}$. Wild-type (WT) C57BL/6 J mice were obtained from CLEA Japan. All experimental protocols were reviewed and approved by the Akita University Institutional Committee for Animal Studies and the Tokyo Medical and Dental University Ethics Committee for Animal Experiments, and all experiments were performed according to their regulations.

Plasmids. EGFP-tagged IpgD in the pEGFP-C1 expression vector was the generous gift of Dr. B. Payrastre (French Institute of Health and Medical Research). FLAG tagged PIK3CA-E545K and PIK3CA-H1047R in the pcDNA3.1 expression vector were the kind gifts of Dr. S. Takasuga (Akita University). Plasmids were amplified in DH5a competent cells, and all inserts were confirmed by sequencing.

Cell culture and transfection. HEK293T, PC3, LNCap and HeLa cells (ATCC) were maintained in DMEM (high glucose, Nacalai Tesque) supplemented with $0.1 \mathrm{mg} / \mathrm{mL}$ streptomycin, 100 units $/ \mathrm{mL}$ penicillin, and $10 \%$ fetal bovine serum (FBS; Hyclone) at $37^{\circ} \mathrm{C}\left(5 \% \mathrm{CO}_{2}\right.$, humidified atmosphere). HEK293T cells $\left(3 \times 10^{6}\right)$ seeded in $100 \mathrm{~mm}$ dishes were transfected in $8 \mathrm{~mL}$ Opti-MEM (Gibco) using $10 \mu \mathrm{g}$ plasmid DNA and $30 \mu \mathrm{L}$ PEI Max (Polysciences) according to the manufacturer's instructions. After $4 \mathrm{~h}$ of transfection, the medium was changed to fresh DMEM containing $10 \%$ FBS.

Cell and tissue sample preparation. Cells were harvested using a cell scraper and collected into phosphate-buffered saline (PBS; $137 \mathrm{mM} \mathrm{NaCl}, 10 \mathrm{mM} \mathrm{Na} \mathrm{HPO}_{4}$, $\left.2.7 \mathrm{mM} \mathrm{KCl}, 1.8 \mathrm{mM} \mathrm{KH}_{2} \mathrm{PO}_{4}, \mathrm{pH} 7.4\right)$ transferred to a glass tube, and centrifuged at $1000 \times g$ for $5 \mathrm{~min}$ to pellet cells. Cells $\left(3 \times 10^{6}\right)$ were resuspended in $1.5 \mathrm{~mL}$ methanol and subjected to lipid extraction (see below). For mouse prostate tissues, $20 \mathrm{mg}$ tissue was transferred to a Lysing Matrix D tube (MP Bio) in $200 \mu \mathrm{l}$ methanol. The tissue was homogenized using a FastPrep-24TM 5 G (MP Bio), transferred to a glass tube containing $1.5 \mathrm{~mL}$ methanol, and subjected to lipid extraction.

Preparation of conditioned medium. Cells were grown to $60-70 \%$ confluence, whereupon the culture medium was replaced with serum-free medium (DMEM, $0.1 \%$ BSA, $10 \mathrm{mM}$ HEPES, pH7.4). After $20 \mathrm{~h}$ incubation, culture supernatants were collected in $15 \mathrm{~mL}$ tubes, centrifuged at $5000 \times \mathrm{g}$ for $5 \mathrm{~min}$, and filtered through $0.45 \mu \mathrm{m}$ cellulose acetate disks (DISMIC) to remove debris. The filtrate (conditioned medium; CM) was frozen in liquid nitrogen and stored at $-80^{\circ} \mathrm{C}$. For analysis of extracellular phosphoinositides, filtrate $(1 \mathrm{~mL})$ was carefully thawed, mixed with $1.5 \mathrm{~mL}$ methanol, and subjected to lipid extraction.

Preparation of mouse plasma. Plasma was prepared from the blood of anesthetized mice according to a standard protocol ${ }^{23}$. Briefly, C57BL/6 J mice were fasted for $6 \mathrm{~h}$ followed by intraperitoneal injection of PBS (control) or $50 \mathrm{mg} / \mathrm{kg}$ lipopolysaccharide (LPS). Blood was collected at $2 \mathrm{~h}$ post-injection and plasma was obtained by centrifugation at $6000 \times g$ for $10 \mathrm{~min}$. Plasma $(100 \mu \mathrm{L})$ was mixed with $1.5 \mathrm{~mL}$ methanol and subjected to lipid extraction.

Preparation of exosomes. Cells $\left(5 \times 10^{6}\right)$ were seeded in $150-\mathrm{mm}$ culture dishes and cultured for $24 \mathrm{~h}$. After two washes of the dishes with PBS, RPMI containing $1 \%$ exosome-depleted FBS was added. After $48 \mathrm{~h}$ incubation, culture supernatants were centrifuged at $2000 \times g$ for $10 \mathrm{~min}$ at $4{ }^{\circ} \mathrm{C}$ and then passed through a $0.22-\mu \mathrm{m}$ filter (Millipore., Bedford, MA, USA) to remove cells and cellular debris. Filtered supernatants were ultracentrifuged at $110,000 \times \mathrm{g}$ for $70 \mathrm{~min}$ at $4{ }^{\circ} \mathrm{C}(\mathrm{SW} 41 \mathrm{Ti}$ rotor Beckman Coulter), and the pellets were washed with trehalose-containing HEPES buffer (20 mM HEPES pH7.4, $25 \mathrm{mM}$ trehalose) to prevent the aggregation of exosomes. Resuspended pellets were re-ultracentrifuged at $110,000 \times g$ for $70 \mathrm{~min}$ at $4{ }^{\circ} \mathrm{C}$, and finally resuspended in HEPES buffer (20 mM HEPES, pH7.4). The size distribution and concentration of exosomes were determined by nanoparticle tracking analysis (NTA) performed using a NanoSight LM10 instrument (Malvern, Worcestershire, UK). Exosomes $\left(1 \times 10^{10}\right.$ particles $)$ were mixed with $1.5 \mathrm{~mL}$ methanol and subjected to lipid extraction.

Lipid extraction. A given biological sample in $1.5 \mathrm{~mL}$ methanol prepared as described above was mixed with $50 \mu \mathrm{L}$ of a methanol/chloroform (9/1) solution containing $1 \mathrm{nmol} \mathrm{C8:0/C8:0} \mathrm{PI}(4,5) \mathrm{P}_{2}$ (as an absorption inhibitor) and $10 \mathrm{pmol}$ each of synthetic C17:0/C20:4 phosphoinositides $\left[\mathrm{PI}(3,4,5) \mathrm{P}_{3}, \mathrm{PI}(3,4) \mathrm{P}_{2}, \mathrm{PI}(3,5) \mathrm{P}_{2}\right.$, $\mathrm{PI}(4,5) \mathrm{P}_{2}, \mathrm{PI} 3 \mathrm{P}, \mathrm{PI} 4 \mathrm{P}, \mathrm{PI} \mathrm{P}$, and $\left.\mathrm{PI}\right]$ as internal standards. Ultrapure water $(750 \mu \mathrm{L}), 2 \mathrm{M} \mathrm{HCl}(750 \mu \mathrm{L})$, and $1 \mathrm{M} \mathrm{NaCl}(200 \mu \mathrm{L})$ were added to this mixture. After vigorous vortex-mixing, $3 \mathrm{~mL} \mathrm{CHCl}_{3}$ was added followed by further 
vortexing for $2 \mathrm{~min}$. After centrifugation at $1200 \times \mathrm{g}$ for $4 \mathrm{~min}$ at room temperature, the lower organic phase (crude lipid extract) was collected and transferred to a new glass tube.

Pre-concentration of phosphoinositides. DEAE Sepharose Fast Flow (10\% slurry) was rinsed twice with an equal volume of water, once with $1 \mathrm{M} \mathrm{HCl}$, twice with water, once with $1 \mathrm{M} \mathrm{NaOH}$, and twice with water. The resin was resuspended in methanol (50\% slurry) and a $0.5 \mathrm{~mL}$ bed volume was packed in a Pasteur pipette (IWAKI) plugged with glass wool. The column was then equilibrated with chloroform. Crude lipid extract $(2.9 \mathrm{~mL})$ was mixed with $1.5 \mathrm{~mL}$ methanol and applied to the column, washed with $3 \mathrm{~mL}$ chloroform/methanol (1/1) and $2 \mathrm{~mL}$ chloroform/methanol/28\% aqueous ammonia/glacial acetic acid (200/100/3/0.9), followed by elution with $1.5 \mathrm{~mL}$ chloroform/methanol/12 M hydrochloric acid/ ultrapure water (12/12/1/1). The eluate was mixed with $850 \mu \mathrm{L} 120 \mathrm{mM} \mathrm{NaCl}$, centrifuged at $1200 \times g$ for $4 \mathrm{~min}$ at room temperature, and the resultant lower phase (purified phosphoinositides) was collected into a fresh glass tube.

Methylation reaction. Purified phosphoinositides were derivatized by methylation using the method of Clark et al. ${ }^{7}$. Briefly, $150 \mu \mathrm{L} 0.6 \mathrm{M}$ trimethylsilyl diazomethane was added to the purified phosphoinositide fraction prepared as above at room temperature. After $10 \mathrm{~min}$, the reaction was quenched with $20 \mu \mathrm{L}$ of glacial acetic acid. The samples were mixed with $700 \mu \mathrm{L}$ methanol/ultrapure water/chloroform (48/47/3) followed by vortexing for $1 \mathrm{~min}$. After centrifugation at $1200 \times \mathrm{g}$ for $4 \mathrm{~min}$, the lower phase was taken to dryness under a stream of nitrogen and redissolved in $100 \mu \mathrm{L}$ acetonitrile.

\section{Chiral column chromatography and tandem mass spectrometry. PRMC-MS} was performed using a triple quadruple mass spectrometer QTRAP6500 (ABSciex) and a Nexera X2 HPLC system (Shimadzu) combined with a PAL HTC-xt (CTC Analytics) autosampler. The mass range of the instrument was set at $m / z 5-2000$. Spectra were recorded in the positive ion mode as $\left[\mathrm{M}+\mathrm{NH}_{4}\right]^{+}$ions, and the scan duration of MS and MS/MS was $0.5 \mathrm{sec}$. The ion spray voltage was set at $5.5 \mathrm{kV}$, cone voltage at $30 \mathrm{~V}$, and source block temperature at $100{ }^{\circ} \mathrm{C}$. Curtain gas was set at $20 \mathrm{psi}$, collision gas at $9 \mathrm{psi}$, ion source gas pressures $1 / 2$ at $50 \mathrm{psi}$, declustering potential at $100 \mathrm{~V}$, entrance potential at $10 \mathrm{~V}$, and collision cell exit potential at $12 \mathrm{~V}$. Collision energy values for each acyl variant are listed in Supplementary Table 1. Lipid sample $(10 \mu \mathrm{L})$ was injected using the autosampler, and molecules were separated using a CHIRALPAK IC-3 column [cellulose tris(3,5-dichlorophenylcarbamate), $2.1 \mathrm{~mm} \varphi \times 250 \mathrm{~mm}, 3 \mathrm{~mm}$, DAICEL] at $22^{\circ} \mathrm{C}$. LC was operated at a flow rate of $100 \mu \mathrm{L} / \mathrm{min}$ with a gradient as follows: $40 \%$ mobile phase A (methanol/5 $\mathrm{mM}$ ammonium acetate) and $60 \%$ mobile phase $\mathrm{B}$ (acetonitrile/5 mM ammonium acetate) were held for $1 \mathrm{~min}$, linearly increased to $85 \%$ mobile phase A over $2 \mathrm{~min}$, and held at $85 \%$ mobile phase A for $11 \mathrm{~min}$. The column was reequilibrated to $40 \%$ mobile phase A for $6 \mathrm{~min}$ before the next injection. Analyst 1.6.3 (SCIEX) was used for data acquisition and processing. MultiQuant (SCIEX) was used for manual data evaluation for peak integration. No background subtraction was performed. Gaussian smoothing width was 1.0 points. For quality control, the peaks from a sample run in which the cps of the surrogate internal standards (SIS; C37:4 phosphoinositides) from the MRM scan below $2 \times 10^{4}$ were not subjected to quantification analysis. The sample peak area value (equivalent to $1 \times 10^{6}$ cells, $10 \mathrm{mg}$ tissue, $1 \times 10^{10}$ exosome particles, or $1 \mathrm{ml}$ plasma) was divided by the corresponding SIS peak area value (equivalent to $1 \mathrm{pmol}$ ) for relative quantification.

Reverse-phase column chromatography-tandem mass spectrometry. C-MS/ MS was performed with an LC system [UltiMate 3000 (Thermo Fisher Scientific) and HTC PAL autosampler (CTC Analytics)] connected to a triple-stage quadrupole mass spectrometer (TSQ Vantage, Thermo Fisher) as described previously ${ }^{24}$. Briefly, methylated lipids were separated on a C18 column (GL Sciences) using a solvent gradient as follows: $0-1 \mathrm{~min}$ hold $70 \% \mathrm{~A} / 30 \% \mathrm{~B}, 1-3 \mathrm{~min}$ constant gradient to reach $90 \% \mathrm{~A} / 10 \% \mathrm{~B}, 3-7.5 \mathrm{~min}$ constant at $90 \% \mathrm{~A} / 10 \% \mathrm{~B}$, 7.5-13 min 30\% A/70\% B, where mobile phase A was acetonitrile/water/70\% ethylamine (800:200:1.3) and mobile phase B was acetonitrile/isopropanol/ $70 \%$ ethylamine (200:800:1.3). Multiple reaction monitoring (MRM) was employed in positive ion mode.

Statistics. Values in each group are shown by mean $\pm \mathrm{SD}$, and the data are also plotted as symbols. Statistical differences between control and test groups were evaluated by the Dunnett's test (Figs. $2 \mathrm{c}$ and $4 \mathrm{a}$ ) or the unpaired Welch's $t$-test (Figs. 2b, 3a, b, and 4e) using GraphPad Prism (GraphPad Software Inc.).

Reporting summary. Further information on research design is available in the Nature Research Reporting Summary linked to this article.

\section{Data availability}

The mass spectrometry data generated in this study have been deposited in the MetaboBank and the MetaboLights databases under accession codes MTBKS201 and MTBLS3180, respectively. Source data are provided with this paper.
Received: 4 November 2020; Accepted: 2 December 2021; Published online: 10 January 2022

\section{References}

1. Balla, T. Phosphoinositides: tiny lipids with giant impact on cell regulation. Physiol. Rev. 93, 1019-1137 (2013).

2. Sasaki, T. et al. Mammalian phosphoinositide kinases and phosphatases. Prog Lipid Res. 48, 307-343 (2009).

3. Hammond, G. R. \& Balla, T. Polyphosphoinositide binding domains: key to inositol lipid biology. Biochim. Biophys. Acta 1851, 746-758 (2015).

4. Goncalves, M. D., Hopkins, B. D. \& Cantley, L. C. Phosphatidylinositol 3-kinase, growth disorders, and cancer. N. Engl. J. Med. 379, 2052-2062 (2018).

5. Fruman, D. A. et al. The PI3K pathway in human disease. Cell 170, 605-635 (2017).

6. Serunian, L. A., Auger, K. R. \& Cantley, L. C. Identification and quantification of polyphosphoinositides produced in response to platelet-derived growth factor stimulation. Methods Enzymol. 198, 78-87 (1991).

7. Clark, J. et al. Quantification of PtdInsP3 molecular species in cells and tissues by mass spectrometry. Nat. Methods 8, 267-272 (2011).

8. Ghosh, A., Sharma, S., Shinde, D., Ramya, V. \& Raghu, P. A novel mass assay to measure phosphatidylinositol-5-phosphate from cells and tissues. Biosci. Rep. 39, BSR20192502 (2019).

9. Barneda, D., Cosulich, S., Stephens, L. \& Hawkins, P. How is the acyl chain composition of phosphoinositides created and does it matter? Biochem. Soc. Trans. 47, 1291-1305 (2019).

10. Ikeda, K., Shimizu, T. \& Taguchi, R. Targeted analysis of ganglioside and sulfatide molecular species by LC/ESI-MS/MS with theoretically expanded multiple reaction monitoring. J. Lipid Res. 49, 2678-2689 (2008).

11. Cai, T. et al. Profiling and relative quantitation of phosphoinositides by multiple precursor ion scanning based on phosphate methylation and isotopic labeling. Anal. Chem. 87, 513-521 (2015).

12. Niebuhr, K. et al. Conversion of PtdIns $(4,5) \mathrm{P}(2)$ into PtdIns(5)P by the S.flexneri effector IpgD reorganizes host cell morphology. Embo J. 21, 5069-5078 (2002).

13. Van der Kaay, J., Beck, M., Gray, A. \& Downes, C. P. Distinct phosphatidylinositol 3-kinase lipid products accumulate upon oxidative and osmotic stress and lead to different cellular responses. J. Biol. Chem. 274, 35963-35968 (1999)

14. Boukhalfa, A. et al. PI3KC2alpha-dependent and VPS34-independent generation of PI3P controls primary cilium-mediated autophagy in response to shear stress. Nat. Commun. 11, 294 (2020).

15. Dowdle, W. E. et al. Selective VPS34 inhibitor blocks autophagy and uncovers a role for NCOA4 in ferritin degradation and iron homeostasis in vivo. Nat. Cell Biol. 16, 1069-1079 (2014).

16. Malek, M. et al. PTEN regulates $\mathrm{PI}(3,4) \mathrm{P} 2$ signaling downstream of class I PI3K. Mol. Cell 68, 566-580 e510 (2017).

17. Scott, W. J. et al. Discovery and SAR of novel 2,3-dihydroimidazo[1,2-c] quinazoline PI3K inhibitors: identification of copanlisib (BAY 80-6946). ChemMedChem 11, 1517-1530 (2016)

18. Suzuki, A., Nakano, T., Mak, T. W. \& Sasaki, T. Portrait of PTEN: messages from mutant mice. Cancer Sci. 99, 209-213 (2008).

19. Wu, X. et al. Generation of a prostate epithelial cell-specific Cre transgenic mouse model for tissue-specific gene ablation. Mech. Dev. 101, 61-69 (2001)

20. Suzuki, A. et al. T cell-specific loss of Pten leads to defects in central and peripheral tolerance. Immunity 14, 523-534 (2001).

21. Li, P. \& Lämmerhofer, M. Isomer selective comprehensive lipidomics analysis of phosphoinositides in biological samples by liquid chromatography with data independent acquisition tandem mass spectrometry. Anal. Chem. 93, 9583-9592 (2021)

22. Hammond, G. R. V. \& Burke, J. E. Novel roles of phosphoinositides in signaling, lipid transport, and disease. Curr. Opin. Cell Biol. 63, 57-67 (2020)

23. Lukka, P. B., Paxton, J. W., Atwell, G. J., Kestell, P. \& Baguley, B. C. A rapid LC-MS/MS method for the quantitation of a series of benzonaphthyridine derivatives: application to in vivo pharmacokinetic and lipophilicity studies in drug development. J. Pharm. Biomed. Anal. 63, 9-16 (2012).

24. Koizumi, A. et al. Increased fatty acyl saturation of phosphatidylinositol phosphates in prostate cancer progression. Sci. Rep. 9, 13257 (2019).

\section{Acknowledgements}

We thank all the present and former members of our laboratories for technical support and constructive suggestions for this project. We also thank Drs. T. Shimizu, P. Hawkins, L. Stephens, J. Clark, T. Habuchi, and T. Takenawa for inspiring discussions. This work was supported by Grants-in-Aid from JSPS (26670132, 15H04693, $20 \mathrm{H} 04920$ to J.S., and $26253006,15 \mathrm{H} 05899,17 \mathrm{H} 03980,20 \mathrm{H} 03433$ to T.S.) and AMED (18gm0710002h0006 to T.S.) and Takeda Science Foundation (to J.S.) 


\section{Author contributions}

T.S., J.S., and A.S. conceived and supervised the project. S.M. and H.N. conducted mass spectrometry and biological experiments and analyzed the data. S.M., T.Y., J.H., E.T., T.Sak., Y.K., M.Y., and J.S. performed biological experiments and analyzed the data. T.H. and C.O. analyzed exosomes. All authors interpreted data, discussed results, and contributed to writing the manuscript. Correspondence and materials should be addressed to J.S. and T.S.

\section{Competing interests}

H.N., J.S., and T.S. have filed for a patent (appl. No. 2018-528909), related to data in this paper on the phosphoinositide measurement method, which is under review. H.N serves as the CEO of and holds equity in LIPIDOME LAB Co., Ltd. T.S. is the founder of Japan Lipid Technologies, LLC. T.Y. serves as the CEO of Japan Lipid Technologies, LLC. The remaining authors declare no competing interests.

\section{Additional information}

Supplementary information The online version contains supplementary material available at https://doi.org/10.1038/s41467-021-27648-z.

Correspondence and requests for materials should be addressed to Junko Sasaki or Takehiko Sasaki.
Peer review information Nature Communications thanks Bart Vanhaesebroeck, and the other, anonymous, reviewer(s) for their contribution to the peer review of this work.

Reprints and permission information is available at http://www.nature.com/reprints

Publisher's note Springer Nature remains neutral with regard to jurisdictional claims in published maps and institutional affiliations.

\begin{abstract}
cc (i) Open Access This article is licensed under a Creative Commons Attribution 4.0 International License, which permits use, sharing, adaptation, distribution and reproduction in any medium or format, as long as you give appropriate credit to the original author(s) and the source, provide a link to the Creative Commons license, and indicate if changes were made. The images or other third party material in this article are included in the article's Creative Commons license, unless indicated otherwise in a credit line to the material. If material is not included in the article's Creative Commons license and your intended use is not permitted by statutory regulation or exceeds the permitted use, you will need to obtain permission directly from the copyright holder. To view a copy of this license, visit http://creativecommons.org/ licenses/by/4.0/.
\end{abstract}

(C) The Author(s) 2022 